\title{
Cardiovascular Complications of COVID-19: Pharmacotherapy Perspective
}

\author{
Azita Hajhossein Talasaz ${ }^{1,2}$ (D) Hessam Kakavand ${ }^{2} \cdot$ Benjamin Van Tassell $^{3,4} \cdot$ Maryam Aghakouchakzadeh $^{2}$. \\ Parham Sadeghipour ${ }^{5}$ Steven Dunn ${ }^{6} \cdot$ Babak Geraiely $^{7}$
}

Accepted: 8 July 2020 / Published online: 15 July 2020

(C) Springer Science+Business Media, LLC, part of Springer Nature 2020

\begin{abstract}
Coronavirus disease of 2019 (COVID-19), which is caused by severe acute respiratory syndrome coronavirus 2 (SARS-CoV-2), is spreading rapidly the world over. The disease was declared "pandemic" by the World Health Organization. An approved therapy for patients with COVID-19 has yet to emerge; however, there are some medications used in the treatment of SARSCoV-2 infection globally including hydroxychloroquine, remdesivir, dexamethasone, protease inhibitors, and anti-inflammatory agents. Patients with underlying cardiovascular disease are at increased risk of mortality and morbidity from COVID-19. Moreover, patients with chronic stable states and even otherwise healthy individuals might sustain acute cardiovascular problems due to COVID-19 infection. This article seeks to review the latest evidence with a view to explaining possible pharmacotherapies for the cardiovascular complications of COVID-19 including acute coronary syndrome, heart failure, myocarditis, arrhythmias, and venous thromboembolism, as well as possible interactions between these medications and those currently administered (or under evaluation) in the treatment of COVID-19.
\end{abstract}

Keywords COVID-19 · SARS-Cov2 $\cdot$ Cardiovascular $\cdot$ Pharmacotherapy $\cdot$ Venous thromboembolism $\cdot$ Drug-drug interactions

Azita Hajhossein Talasaz

a-talasaz@tums.ac.ir

1 Tehran Heart Center, Tehran University of Medical Sciences, Tehran Iran

2 Department of Clinical Pharmacy, Tehran University of Medical Sciences, Tehran Iran

3 Department of Pharmacotherapy and Outcome Science, School of Pharmacy, Virginia Commonwealth University, Richmond VA USA

4 Pauley Heart Center, Division of Cardiology, Department of Internal Medicine, School of Pharmacy, Virginia Commonwealth University, Richmond VA USA

5 Cardiovascular Intervention Research Center, Rajaie Cardiovascular Medical and Research Center, Iran University of Medical Sciences, Tehran Iran

6 University of Virginia Health System, Charlottesville VA USA

7 Department of Cardiology, Imam Khomeini Hospital Complex, Tehran University of Medical Sciences, Tehran Iran

\section{Introduction}

Severe acute respiratory syndrome coronavirus 2 (SARS-CoV-2), which can lead to coronavirus disease of 2019 (COVID-19), is spreading rapidly the world over. At the time of writing this article (July 5, 2020), it has infected over 11 million people and caused more than 500,000 deaths globally (https://www.who.int/docs/ default-source/coronaviruse/situation-reports/20200705covid-19-sitrep-167.pdf?sfvrsn=17e7e3df_4). The disease was declared "pandemic" by the World Health Organization (WHO) on March 11, 2020 [1].

SARS-CoV-2 is a single-strand RNA virus from the Coronaviridae family; it can affect various organs including, but not limited to, the respiratory system, the kidneys, the gastrointestinal system, and the cardiovascular system [2]. Data published from Wuhan, China indicate that patients with underlying cardiovascular disease (CVD) are at increased risk of mortality and morbidity from COVID-19 [3]. This infectious disease can affect the cardiovascular system, both directly and indirectly [4]. The increased susceptibly of patients with CVD in the COVID-19 outbreak notwithstanding, the fact that this population is already under treatment with 
various classes of medication places them at higher risk of adverse drug events and possible drug-drug interactions.

Although our current understanding of COVID-19 pathology would suggest an increased number of cardiovascular complications during the pandemic, the total number of cardiovascular-related admissions has paradoxically declined by $43 \%$ in the United States [5]. However, this may be largely attributable to the fear of contracting COVID-19 at medical centers, and thus not indicative of a true reduction in cardiovascular diseases. It is prudent, therefore, to pay sufficient heed to the cardiovascular problems of our patients even more than before, as it is still the number one killer globally, even in the COVID-19 era.

An approved therapy for patients with COVID-19 has yet to emerge; however, there are some medications used in the treatment of SARS-CoV-2 infection globally. They include hydroxychloroquine/chloroquine, which has immunomodulatory effects and can inhibit viral fusion; lopinavir/ritonavir, which is an antiretroviral agent and can block virus replication; dexamethasone as an anti-inflammatory agent; remdesivir, which is an inhibitor of RNA synthesis; and lastly passive immunotherapeutic agents such as tocilizumab, sarilumab, canakinumab, and anakinra [6]. Although not yet approved by any regulatory agencies, remdesivir has shown evidence of shortened time to recovery among patients with COVID-19 [7]. According to the recent RECOVERY trial, dexamethasone showed beneficial effects in patients after 7 days of treatment initiation at a dose of $6 \mathrm{mg} /$ day for up to 10 days, as it reduced 28-day mortality in patients under mechanical ventilation by one third, and by one fifth in patients only on supplemental oxygen [8]. Finally, convalescent plasma showed positive effects by improving the rate of nasopharyngeal viral RNA clearance at $72 \mathrm{~h}$ in patients with severe but not life-threatening COVID-19. However, it did not increase the overall rate of clinical improvement in patients with lifethreatening COVID-19 infection [9].

This article seeks to review the most up-to-the-minute evidence with a view to explaining possible pharmacotherapies for the cardiovascular complications of COVID-19 including acute coronary syndrome (ACS), heart failure, myocarditis, arrhythmias, and coagulopathy, as well as possible interactions between these medications and those currently administered (or under evaluation) in the treatment of COVID-19.

\section{SARS-CoV-2 and the Cardiovascular System}

Similar to other coronaviruses, SARS-CoV-2 uses angiotensin-converting enzyme 2 (ACE2) for cell entry [10]. ACE2 is a membrane protein highly expressed in the lung, enterocytes, and the heart and is responsible for converting angiotensin II to angiotensin-(1-7), which is a vasodilator peptide with a potential role in lung protection [11].
Based on animal studies, SARS-CoV-2 can downregulate ACE2 expression, facilitate neutrophil infiltration, and subsequently accelerate lung and myocardial damage [12]. Such damage is speculated to be more profound in patients with cardiovascular disorders because they may be more likely to have upregulated ACE2 expression due to the pharmacological inhibitors of the renin-angiotensin-aldosterone system (RAAS) and underlying conditions [13, 14]. SARS-CoV-2 infections can also directly lead to viral myocarditis and elevated levels of myocardial injury markers, troponin, and creatine kinase [15]. In severe cases, SARS-CoV-2 disease progresses from ACE2-dependent alveolar damage and hypoxia to systemic inflammatory response syndrome (acute respiratory distress syndrome [ARDS]) and an exaggerated cytokine release. The result may be myocardial supply-and-demand imbalance, plaque rupture, and thrombosis because of procoagulant states (Fig. 1).

These hypotheses may explain not only why patients with CVD are prone to higher rates of SARS-CoV-2 infections and mortality, but also how patients with chronic stable states and even otherwise healthy individuals might sustain acute cardiovascular problems during the COVID-19 pandemic.

\section{Acute Coronary Syndrome}

Based on different studies from China, the incidence of acute cardiac injury, defined as an elevation in hypersensitive troponin, currently ranges from $7.2 \%$ to $19.7 \%$ in patients with COVID-19 [16-18]. Mildly increased cardiac troponin levels (e.g., $<2-3$ times the upper limits of normal), particularly in the elderly with pre-existing cardiac diseases, do not need workup or treatment for type1 myocardial infarction unless it is suggested by angina symptoms and/or electrocardiography (ECG) changes. Minimal elevations in troponin levels can be explained by pre-existing cardiac diseases or myocardial injury due to SARS-CoV-2 [19]. Consequently, changes in biomarkers, patient presentation, past medical history, and ECG changes should be considered altogether. Indeed, efforts should be concentrated on the differentiation of viral-related cardiac injury from ACS. It is in this context that the limitations of primary percutaneous coronary interventions in the current pandemic era have conferred on pharmacotherapy a more crucial role than ever before [20].

Antiplatelets An effective antiplatelet regimen is a matter of utmost importance in ACS management. The optimal antiplatelet regimen hinges on the treatment strategy and the COVID-19 status. The current guidelines stipulate that if fibrinolytic agents are to be used in patients not receiving active therapy for COVID-19, the $\mathrm{P}_{2} \mathrm{Y}_{12}$ inhibitor of choice is clopidogrel [21], although ticagrelor can also be considered according to the TREAT trial [22]. In patients undergoing 


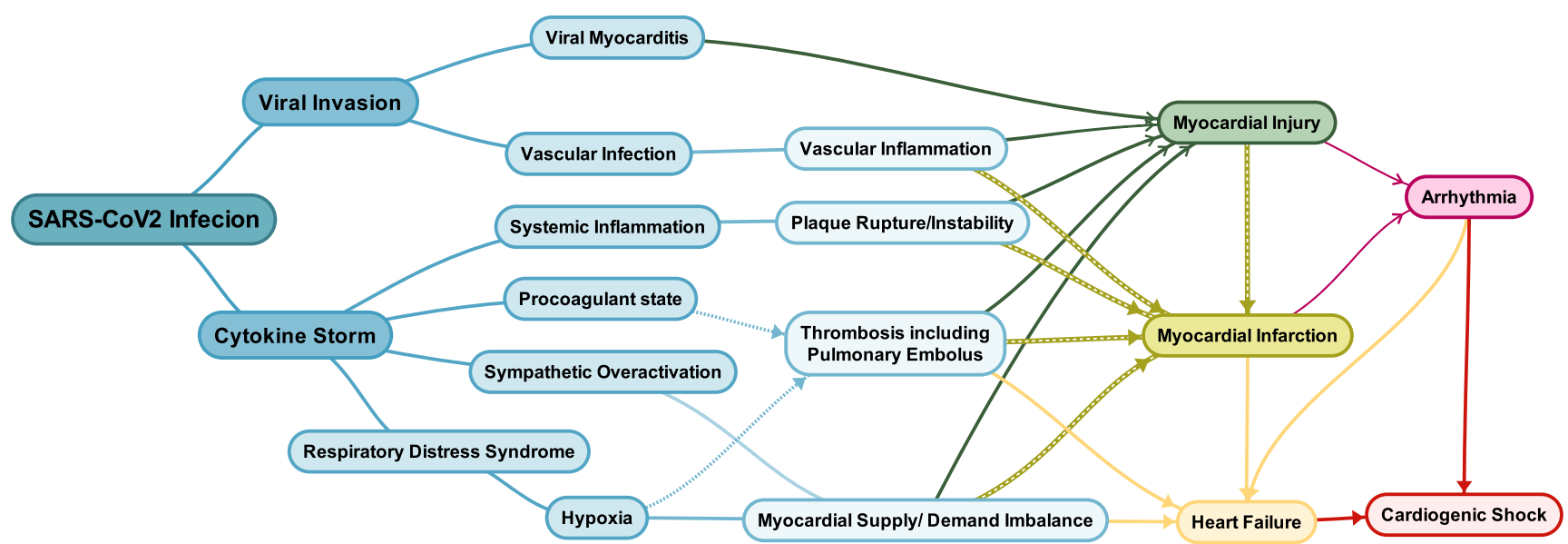

Fig. 1 Cardiovascular complications of COVID-19 are illustrated herein

primary percutaneous interventions, prasugrel and ticagrelor are recommended. Whatever the case may be, decisionmaking with regard to patients with COVID-19 should be in light of drug-drug and drug-disease interactions. Aspirin at the low dose administered in ACS has minimal antiinflammatory effects, so concerns regarding the potentially detrimental effects of nonsteroidal anti-inflammatory drugs on the clinical course of COVID-19 are not applicable to aspirin [19]. The loading and maintenance doses of aspirin for patients with COVID-19 should be the same as those administered to the normal population. If the patient has coagulopathy, clopidogrel is the $\mathrm{P}_{2} \mathrm{Y}_{12}$ inhibitor of choice because it poses the lowest bleeding risk [21]. Another concern over the prescription of antiplatelets is the interaction between $\mathrm{P}_{2} \mathrm{Y}_{12}$ inhibitors and protease inhibitors. Lopinavir/ritonavir is a potential antiviral agent for COVID-19; it can, through the strong inhibition of the CYP3A4 pathway, increase the risk of bleeding with ticagrelor. Consequently, ticagrelor is contraindicated in patients receiving lopinavir/ritonavir. Lopinavir/ ritonavir could also diminish the antiplatelet activity of clopidogrel, which is why the platelet function test is suggested to adjust the clopidogrel dose accordingly. In individuals without coagulopathy and bleeding risk, prasugrel may be a good option while lopinavir/ritonavir is administered (Table 1) [23]. With respect to glycoprotein IIb/IIIa inhibitors, given the high bleeding risk and propensity for acute kidney injury in patients with COVID-19, their use should ideally be avoided. Tirofiban and eptifibatide may be considered if needed, but abciximab is not recommended due to the high risk of thrombocytopenia [21].

Anticoagulants In a large cohort study on patients with COVID-19, Haung et al. [16] recorded 5\% thrombocytopenia (platelet count $<100,000 / \mathrm{L}$ ) in the entire population, with a higher prevalence among patients in the ICU (8\%). As low molecular weight heparin (LMWH) may have advantages over unfractionated heparin (UFH) in patients with coagulopathy [24], it may be regarded as the agent of choice. Still, if the patient is considered at high bleeding risk or has severe kidney injury, UFH may be preferable, because protamine is available for its reversal if bleeding occurs. In patients receiving fibrinolytic therapy, LMWH can be used as the agent of choice if the cardiologist defers angiography [21]. Nonetheless, in centers where angiography is routinely done within $24 \mathrm{~h}$ of fibrinolytic therapy, UFH still constitutes the best option (Table 1). If complete revascularization is achieved per the typical management of these patients, in the absence of risk factors for vein thrombosis, anticoagulants can be discontinued [21]. It should be noted that there are no interactions between parenteral antithrombotic agents and the currently available COVID-19 medications [23].

Thrombolytics Reperfusion therapy with thrombolytics is an attractive option in stable patients without contraindications. Tenecteplase, the most fibrin-specific agent, is a prudent choice in patients with COVID-19 on the strength of its high success rate, low bleeding risk, and single-dose administration strategy [21]. Tenecteplase is also associated with the least fibrin depletion of all fibrinolytics.

Statins Statins have beneficial vascular and myocardial effects that are attributed to their anti-inflammatory effects, which may explain why the continued use of this class of medication in patients with a cardiovascular or cardiometabolic indication may have positive effects in the presence of acute viral respiratory infections [25]. Irrespective of COVID-19, a highintensity statin (atorvastatin or rosuvastatin) should be administered to every patient with ACS [26]. Cohort studies in China have demonstrated prevalent elevated levels of hepatic transaminase and creatine kinase in those afflicted with COVID-19 [16-18]. It is advisable, therefore, to monitor the liver function and creatine kinase levels at baseline and periodically thereafter. In the case of severe rhabdomyolysis or increased liver enzymes, it is recommended that statins be 
Table 1 Common drug-drug interactions and recommendations for use in patients with COVID-19

\begin{tabular}{|c|c|c|c|}
\hline \multicolumn{2}{|l|}{ Class of drug } & \multirow{2}{*}{$\begin{array}{l}\text { Concerns } \\
\text { Increased risk of bleeding }\end{array}$} & Recommendation \\
\hline \multirow[t]{5}{*}{ Antiplatelets } & P2Y12 inhibitors & & $\begin{array}{l}\text { With fibrinolytics and patients with coagulopathy: } \\
\text { clopidogrel is preferred }\end{array}$ \\
\hline & & $\begin{array}{l}\text { In patients receiving lopinavir/ritonavir, increased risk } \\
\text { of bleeding with ticagrelor, and decreases antiplatelet } \\
\text { activity with clopidogrel }\end{array}$ & Prasugrel is recommended with lopinavir/ritonavir \\
\hline & Glycoprotein IIb/IIIa & Increased risk of bleeding & Their use should be restricted \\
\hline & Inhibitors & & Can be considered if needed \\
\hline & & & Abciximab is not recommended \\
\hline \multirow[t]{11}{*}{ Anticoagulants } & LMWH & Increased risk of bleeding & $\begin{array}{l}\text { In patents with coagulopathy and with fibrinolytic } \\
\text { therapy, LMWH is preferred }\end{array}$ \\
\hline & & & $\begin{array}{l}\text { (If angiography is going to be done within } 24 \mathrm{~h} \\
\text { after fibrinolytic therapy, UFH is still the best option) }\end{array}$ \\
\hline & UFH & Increased risk of bleeding & $\begin{array}{l}\text { If the patient is considered to be at high bleeding } \\
\text { risk, UFH could be considered. }\end{array}$ \\
\hline & Rivaroxaban & $\begin{array}{l}\text { In patients receiving lopinavir/ritonavir, increased } \\
\text { risk of bleeding }\end{array}$ & Contraindicated with lopinavir/ritonavir \\
\hline & & Interaction with tocilizumab & 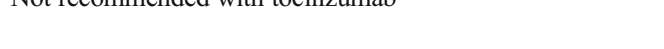 \\
\hline & Apixaban & $\begin{array}{l}\text { In patients receiving lopinavir/ritonavir, } \\
\text { increased risk of bleeding }\end{array}$ & $\begin{array}{l}\text { Contraindicated/reduce dose by } 50 \% \text { with lopinavir/ritonavir } \\
\text { Not recommended with Tocilizumab }\end{array}$ \\
\hline & & Interaction with tocilizumab & \\
\hline & Dabigatran & $\begin{array}{l}\text { In patients receiving lopinavir/ritonavir, } \\
\text { increased risk of bleeding }\end{array}$ & Use not recommended/suggested as an option, data lacking \\
\hline & Edoxaban & $\begin{array}{l}\text { In patients receiving lopinavir/ritonavir, i } \\
\text { ncreased risk of bleeding }\end{array}$ & $\begin{array}{l}\text { Stroke prevention in non-valvular AF: no } \\
\text { dose adjustment }\end{array}$ \\
\hline & & & VTE indication: reduce dose by $50 \%$ \\
\hline & Warfarin & $\begin{array}{l}\text { In patients receiving lopinavir/ritonavir/tocilizumab, } \\
\text { may reduce or increase INR }\end{array}$ & OAC of choice, monitor INR \\
\hline Fibrinolytics & & Increased risk of bleeding & Tenecteplase is preferred \\
\hline \multirow[t]{3}{*}{ Statins } & & Increased risk of hepatotoxicity & Monitor LFT \\
\hline & & Increased effect with lopinavir/ritonavir & Reduce dose with lopinavir/ritonavir \\
\hline & & Decreased effect with tocilizumab & Monitor with tocilizumab \\
\hline \multicolumn{2}{|l|}{ Beta-blockers } & Increased risk of bradycardia, hypotension and AV & Metoprolol and bisoprolol are preferred \\
\hline \multirow{5}{*}{\multicolumn{2}{|c|}{ ACEI/ARB/ARNI }} & block with concomitant use of protease inhibitors & Monitor BP and HR \\
\hline & & Decreased effects of losartan and & Continuation of ACEI/ARB \\
\hline & & irbesartan with lopinavir/ritonavir & Monitor BP, SCr, and $\mathrm{K}$ \\
\hline & & Increased effects of valsartan with lopinavir/ritonavir & \\
\hline & & $\begin{array}{l}\text { Increased concentration of sacubitril } \\
\text { with lopinavir/ritonavir }\end{array}$ & \\
\hline \multirow{3}{*}{\multicolumn{2}{|c|}{ Mineralocorticoid antagonists }} & Increased risk of adverse effect with lopinavir/ritonavir & Spironolactone is preferred \\
\hline & & & Eplerenone is contraindicated \\
\hline & & & Monitor serum electrolytes and $\mathrm{SCr}$ \\
\hline \multirow{2}{*}{\multicolumn{2}{|c|}{ Diuretics }} & $\begin{array}{l}\text { Increase the concentration of indapamide with } \\
\text { lopinavir/ritonavir }\end{array}$ & $\begin{array}{l}\text { Monitor BP, serum electrolytes for prevention } \\
\text { of TdP }\end{array}$ \\
\hline & & & Monitor BUN/SCr \\
\hline \multicolumn{2}{|l|}{ Digoxin } & Increased risk of toxicity & Monitor digoxin concentration \\
\hline \multicolumn{2}{|l|}{ Ivabradine } & Increased risk of adverse effect with lopinavir/ritonavir & Do not co-administer with lopinavir/ritonavir \\
\hline \multirow{6}{*}{\multicolumn{2}{|c|}{ Antiarrhythmics }} & Increased risk of QT prolongation and TdP with & Monitor ECG before initiation \\
\hline & & concomitant use of chloroquine/hydroxychloroquine & Maintain serum $\mathrm{Mg}>2$ and $\mathrm{K}>4 \mathrm{mg} / \mathrm{dL}$ \\
\hline & & Increased antiarrhythmic effects with & Lidocaine and quinidine are preferred. Monitor \\
\hline & & protease inhibitors & ECG and adjust dose if necessary \\
\hline & & & Flecainide and propafenone are not recommended \\
\hline & & & ECG, BP, LFT, and TFT should be monitored \\
\hline
\end{tabular}

ACEI: angiotensin converting enzyme inhibitor; ARB: angiotensin receptor blocker; ARNI: angiotensin receptor neprilysin inhibitor; AF: atrial fibrillation; AV: atrioventricular; BP: blood pressure; BUN: blood urea nitrogen; ECG: electrocardiogram; HR: heart rate; INR: international normalized ratio; LFT: liver function test; LMWH: low molecular weight heparin; OAC: oral anticoagulant; SCr: serum creatinine; TdP: torsade de pointes; TFT: thyroid function test; UFH: unfractionated heparin; VTE: venous thromboembolism

temporarily withheld [19]. Another concern is the potential drug interaction between atorvastatin or rosuvastatin and lopinavir/ritonavir. The respective daily dose of atorvastatin and rosuvastatin should not exceed $20 \mathrm{mg}$ and $10 \mathrm{mg}$ while in concomitant use with lopinavir/ritonavir (Table 1) [27]. Interleukin-6 (IL-6) receptor antibodies such as tocilizumab may also lower statin concentrations by reversing cytochrome P450 (CYP45) suppression; however, the clinical significance 
of this effect may be minimized by the short-term use of antiIL-6 receptor treatments [25].

Beta-Blockers According to the guidelines on ACS, beta-1 selective beta-blockers may be preferred over nonselective agents [21]. Among beta-1 selective beta-blockers, whereas metoprolol is metabolized by CYP2D6 and bisoprolol is partially metabolized by CYP3A4 and CYP2D6, atenolol is not metabolized by CYP450 (Table 1) [28]. Bradycardia with hydroxychloroquine has also been reported, which may be potentiated with beta-blockers. As with patients receiving any beta-blocker, diligent monitoring of the heart rate and blood pressure is warranted; patients with hemodynamic instability should not receive beta-blockers unless there are clear improvements in their blood pressure and heart rate.

Angiotensin-Converting Enzyme Inhibitors (ACEIs) and Angiotensin-Receptor Blockers (ARBs) There is conflicting evidence on the pros and cons of the use of ACEIs and ARBs in patients with COVID-19. Fang et al. [29] demonstrated that ACE2 was upregulated in their patients chronically receiving RAAS blockers, which might place such patients at increased risk of infection with SARS-CoV-2. Conversely, some animal models have indicated the potentially protective effect of losartan administration in the lungs [30]. At present, there are three major studies on ACEIs and ARBs in patients with COVID-19, and they have all reported no harm in the use of RAAS blockers in patients with COVID-19 [31-33]. Given the conflicting reports on this issue, almost all international societies $[34,35]$ have recommended against the discontinuation of ACEIs and ARBs due to COVID-19. Hence, in patients with ACS, an ACEI or ARB should be started in the first $24 \mathrm{~h}$ after admission, as is the case in the normal population [21]. The important issue here is the potential drug interaction between lopinavir/ritonavir and ARBs. When administered concomitantly with ritonavir, losartan and irbesartan may have less ability to transform into their respective active moiety and, as such, lose their efficiency [28]. On the other hand, the concentration of valsartan may increase through the inhibition of organic anion-transporting polypeptide 1B1 (OATP1B) and the hepatic efflux transporter, multidrug resistance-associated protein 2 (MRP20) [28]. Ultimately, in patients with COVID-19 receiving ACEIs and ARBs, the close monitoring of blood pressure, serum creatinine, and potassium is recommended [25].

\section{Heart Failure}

Pump failure can happen due to myocardial injury, and chronic stable heart failure is liable to worsen during COVID-19 infection. Heart failure in patients with COVID-19 can range from mild heart failure with preserved ejection fraction in the early stages of the illness [36] to severe end-stage heart failure and cardiogenic shock with high rates of mortality [37]. The risk of mortality and morbidity is also higher in patients with underlying heart failure [19].

Beta-Blockers With the exclusion of patients with hemodynamic instability and lung edema, patients with heart failure with reduced ejection fraction should receive a beta-blocker. Similar to patients with ACS, beta- 1 selective beta-blockers (i.e., metoprolol succinate and bisoprolol) may be preferred in patients with respiratory infections. Among the beta-blockers used for heart failure, carvedilol is metabolized by glucuronidation (UGT1A1) and CYP450 (primarily 2D6 and 2C9), metoprolol is chiefly metabolized by CYP2D6, and bisoprolol is partially metabolized by CYP3A4 and CYP2D6 (Table 1) [28]. Ritonavir can consequently affect the metabolism of all of these agents, which is why the watchful monitoring of the heart rate and blood pressure of patients receiving lopinavir/ritonavir is highly recommended.

\section{ACEIs/ARBs/Angiotensin Receptor-Neprilysin Inhibitor (ARNIs)} Similar to the ACS scenario, regardless of COVID-19 infection, patients with heart failure with reduced ejection fraction should receive an ACEI or ARB under close surveillance [19]. The concentration of sacubitril rises in tandem with the administration of ritonavir, hence the importance of the thorough monitoring of blood pressure, serum creatinine, and potassium in patients on sacubitril/valsartan (Table 1).

Diuretics There is no specific interaction between loop diuretics and anti-COVID medications, so these drugs can be safely initiated or continued in patients with COVID-19. Given the high rate of acute kidney injury among this group of patients, diuretic therapy should be closely monitored in terms of vital signs, serum electrolytes, and creatinine. Mineralocorticoid antagonists, if administered under close surveillance, can also be continued in patients with COVID19. Concerning patients receiving protease inhibitors, the area under the curve (AUC) of eplerenone can increase fourfold, which may favor the use of spironolactone in patients on lopinavir/ritonavir (Table 1). No specific interaction is known between thiazides and lopinavir/ritonavir, with indapamide being an exception. The concentration of indapamide may increase while administered with lopinavir/ritonavir, necessitating extreme caution. It is also essential to take heed of the risk of QT prolongation associated with chloroquine/ hydroxychloroquine. As patients with electrolyte disturbances are at greater risk, those receiving diuretics should be monitored for potassium and magnesium levels [25].

Digoxin All patients who are on digoxin and hospitalized for COVID-19 should be monitored meticulously because of the possibility of instability during acute infection. This may 
necessitate the measurement of serum concentration in patients with COVID-19 who continue to receive digoxin. Furthermore, ritonavir can increase the AUC of digoxin by $29 \%$ and its halflife by $43 \%$. Hydroxychloroquine can also increase the concentration of digoxin through an unknown mechanism [25]. The narrow therapeutic index, drug-drug interactions, and potential for the unpredictable serum concentrations of digoxin may require physicians to reconsider the continued use of digoxin for patients with COVID-19 (Table 1).

Hydralazine/Nitrates Protease inhibitors can reduce the formation of nitric oxide from isosorbide dinitrate and, as such, compromise its efficacy. Protease inhibitors also significantly interact with hydralazine. Dose adjustments based on the patient's response should be considered [28].

Ivabradine Ivabradine is an I(f) channel blocker used for symptomatic management in patients with ejection fractions of $35 \%$ or lower, sinus rhythms, and minimum resting heart rates of 70 beats per minute despite a maximum tolerable dose of beta-blockers. It appears that ivabradine can be continued safely in hemodynamically stable patients with COVID-19. The coadministration of potent CYP450 inhibitors can increase the AUC of ivabradine 6-fold, so ivabradine should be discontinued in patients receiving lopinavir/ritonavir for COVID-19 [25].

\section{Myocarditis}

Viral infections are the most common etiology of myocarditis and are self-limiting without sequelae in most cases [38]. The pathophysiology of COVID-19 is the same as that of myocarditis in that it is detected several days after fever initiation [39]. The underlying mechanism of myocardial injury in patients with COVID-19 is related to the upregulation of ACE2 in the cardiovascular system $[19,39]$. While a rise in cardiac markers may not be related solely to myocarditis, elevated levels of troponin and brain natriuretic peptide are correlated with worse outcomes $[13,15]$.

It appears that, similar to other instances of viral myocarditis, COVID-19-related myocarditis can exist without clinical symptoms; nevertheless, fulminant viral myocarditis with cardiac enlargement and acute heart failure has also been reported [40]. There is also a case report from Italy that showed endomyocardial localization of the virus in a patient with fulminant myocarditis and cardiogenic shock [41].

Based on the data extracted from cases with myocarditis as a consequence of other viral infections, the treatment of COVID-19-related myocarditis is mostly supportive [38]. Antiviral therapy and immunomodulators can also be of help. The treatment of new-onset heart failure with a balanced use of vasopressors, inotropes, diuretics, vasodilators, and fluid therapy may be lifesaving. Based on the latest guideline on the management of critically ill adults with COVID-19, norepinephrine is the vasopressor of choice, and vasopressin can be added if the target mean arterial pressure is not achieved with norepinephrine. The use of dopamine is discouraged because of the increased risk of arrhythmias and mortality. In patients with COVID-19 who suffer cardiogenic shock, dobutamine is the inotrope of choice, and milrinone can be added in refractory cases [42].

In critically ill patients with COVID-19, the routine use of corticosteroids and intravenous immunoglobulins is not recommended in the absence of ARDS [42, 43]. Corticosteroids may be beneficial to patients with lymphocytic myocarditis [44]. This is also true for patients with COVID-19, as systemic corticosteroids are suggested in mechanically ventilated patients with ARDS [42]. The most significant information on the use of immunomodulators among patients with COVID19 and myocarditis comes from a case report of coronavirus fulminant acute myocarditis, which was treated with methylprednisolone to suppress inflammation $(200 \mathrm{mg}$ /day for 4 days) and immunoglobulin to regulate the immune status (20 g/day for 4 days) [40]. In another case report, a young male patient with COVID-19-related myocarditis recovered without any specific treatment and with just supportive heartfailure-related medications [45]. In the setting of heart failure secondary to ischemia, corticosteroids should be avoided, as they may increase the potential for the mechanical complications of infarction.

SARS-CoV2 can place the patient in the danger of pericardial inflammation due to cytotoxic effects or immunemediated pathways, as reported by Dabbagh et al. [46] Furthermore, colchicine, as a known beneficial agent in the treatment of pericarditis, showed an improvement in the time to clinical deterioration in COVID-19 patients in the GRECCO-19 trail [47].

\section{Arrhythmias}

Cardiac arrhythmias such as tachyarrhythmias and bradyarrhythmias are other cardiovascular complications that occur frequently in patients with COVID-19 [48, 49]. In a study in China, the prevalence of cardiac arrhythmias was higher in ICU patients than in non-ICU patients $(44.4 \%$ vs $6.9 \%)$ [18].

Cardiac arrhythmias may be attributed to direct myocardial injury or secondary to systemic alterations due to acute viremia (e.g., metabolic derangement, hypoxia, neurohormonal changes, and inflammatory stress). Patients with COVID-19 who are in a hyperinflammatory state have hypercytokinemia and arrhythmogenic potential. Cytokine storm and increasing levels of IL-1, IL-2, IL-1 $\beta$, IL-6, monocyte chemoattractant protein, and tumor necrosis factor-alpha (TNF- $\alpha$ ) are associated with fatal arrhythmias [50]. These cytokines, particularly 
IL-6, prolong QTc via their direct effect on the ion channels of cardiomyocytes and their indirect effect on the bioavailability of QTc-prolonging medications by inhibiting CYP3A4 and hyper-activating the cardiac sympathetic system [51].

Several COVID-19 pharmacotherapies including chloroquine/hydroxychloroquine (with or without azithromycin) and lopinavir/ritonavir can prolong the QTc interval and increase the risk of drug-induced torsade de pointes (DI-TdP) and druginduced sudden cardiac death (DI-SCD). The risk of DI-SCD can be augmented if these drugs are used in combination with each other (i.e., chloroquine/hydroxychloroquine plus azithromycin with or without lopinavir/ritonavir) $[52,53]$. The largest multinational registry analysis in this regard to date reported that the use of hydroxychloroquine/chloroquine alone or with a macrolide was associated with an increased risk of de novo ventricular arrhythmias and mortality in patients with COVID-19 [54]. Be that as it may, the manuscript was subsequently retracted, casting doubt on the reliability of the data for decision-making. Another important adverse effect is the risk of atrioventricular block associated with lopinavir/ritonavir and chloroquine/hydroxychloroquine. Other altered cardiac functions secondary to the use of chloroquine/hydroxychloroquine include bundle branch block, ventricular tachycardia, and ventricular fibrillation [48].

Several risk factors have been identified that can increase the risk of DI-TdP and DI-SCD. These risk factors include advanced age, female sex, structural heart disease, congenital long-QT syndromes, electrolyte disturbances (e.g., hypokalemia, hypomagnesemia, and hypocalcemia), concomitant use of loop diuretics, hepatic/renal failure, baseline QTc prolongation, concomitant QTc-prolonging medications, fever, sepsis, and inflammatory state [53, 55-58]. Irrespective of the efficacy of hydroxychloroquine (alone or in combination with azithromycin), the concerns regarding DI-TdP and DI-SCD underscore the need for meticulous surveillance and optimization of these risk factors $[53,57]$. To that end, several risk scores, such as the Tisdale score and the pro-QT score, are utilized for the prediction of drug-associated QTc prolongation $[55,56]$.

The significance of the detection of these risk factors before treatment initiation necessitates baseline and periodic 12-lead ECGs. Also of considerable importance is the baseline and periodic monitoring of the hepatic/renal function, serum potassium, and serum magnesium. All other unnecessary QTcprolonging medications should be discontinued or switched to alternatives with less QTc liability [52, 53, 57].

A recent study posited that a baseline QTc of greater than $500 \mathrm{~ms}$ (or $>530-550 \mathrm{~ms}$ if QRS $>120 \mathrm{~ms}$ ) or a minimum Tisdale risk score of 11 with failure to monitor serial ECGs for inpatients should be regarded as a contraindication to the use of hydroxychloroquine. Moreover, a baseline QTc of greater than $480 \mathrm{~ms}$ (or $>510-530 \mathrm{~ms}$ if QRS $>120 \mathrm{~ms}$ ) or a minimum Tisdale risk score of 11 for outpatients is an absolute or relative contraindication for the combined use of hydroxychloroquine and azithromycin. If the decision has been made to treat a patient with a minimum baseline QTc of $500 \mathrm{~ms}$ with these QTcprolonging medications, it may be reasonable to start with hydroxychloroquine monotherapy $[53,57]$. The discontinuation of azithromycin and/or reduction of the dose of hydroxychloroquine should be considered for patients with an increase in QTc of greater than 30 to $60 \mathrm{~ms}$ after treatment initiation. ECG monitoring should be conducted 2 to $4 \mathrm{~h}$ after the first dose and 48 and $96 \mathrm{~h}$ thereafter for patients with a baseline QTc of greater than $500 \mathrm{~ms}$. For patients with a baseline QTc of less than $500 \mathrm{~ms}$, there is no need to acquire ECG after the first dose. Daily ECGs should be considered for all inpatients receiving QTc-prolonging agents $[53,57,59,60]$. However, the measurement of QTc alone cannot prevent chloroquine- and hydroxychloroquine-induced arrhythmias, since these agents may affect conduction velocity and calcium hemostasis. These changes cannot be assessed by QTc assessment [61].

Another alteration in the ECG of patients with COVID-19 is Brugada-like ECG morphology [62]. It is necessary to differentiate between Brugada syndrome and Brugada-like ECG morphology. Since fever in patients with Brugada syndrome is associated with life-threatening arrhythmic events, these patients should take precautionary measures (e.g., social distancing) and use aggressive antipyretic therapy to alleviate their fever. ECG monitoring should be considered for those who do not respond to antipyretics with body temperatures exceeding $38.5^{\circ} \mathrm{C}[19,60]$.

Drug interactions between antiarrhythmic medications and antiviral agents (e.g., hydroxychloroquine and lopinavir/ritonavir) are another cause for concern. Antiarrhythmic agents can increase the risk of QT prolongation and TdP with the concomitant use of chloroquine/hydroxychloroquine, hence the importance of ECG monitoring before the initiation and maintenance of serum magnesium above $2 \mathrm{mg} / \mathrm{dL}$ and serum potassium above $4.5 \mathrm{mg} / \mathrm{dL}[19,48,52,53]$.

Another source of concern is the interaction between antiarrhythmics and protease inhibitors. Protease inhibitors can augment the effects of amiodarone, lidocaine, and quinidine. In patients for whom the administration of the mentioned antiarrhythmics is necessary, caution should be exercised through ECG monitoring and dose reduction. Additionally, if these antiarrhythmics are in concomitant use with amiodarone, blood pressure and liver/thyroid function should be monitored periodically, and alternative agents should be considered. The coadministration of protease inhibitors and class $1 \mathrm{C}$ antiarrhythmics, flecainide, and propafenone is not recommended [52].

\section{Venous Thromboembolism (VTE)}

Coagulopathy has been reported as a predictor of poor prognosis in patients with COVID-19 [3]. Primary reports on 
COVID-19 comparing patients who did not survive and those who survived showed that the former group had significantly higher levels of D-dimer and other fibrin degradation products, as well as longer prothrombin and activated partial thromboplastin times (aPTT) [63]. Nonetheless, based on recent publications, coagulopathy in patients with COVID-19 manifests as elevated fibrinogen and D-dimer levels, with minimal alterations in the prothrombin time, aPTT, and platelet count in the early stages of the disease [64]. Tang et al. showed that disseminated intravascular coagulation (DIC) occurred in $71.4 \%$ of the non-survivors versus $0.6 \%$ of the survivors during hospitalization in their study [63]. In contrast, in a report by Klok et al., none of the patients in the intensive care unit (ICU) whose cases were complicated by thrombotic events developed DIC [65]. Interestingly, Zhang et al. described three patients with COVID-19 who had overt coagulopathy with positive antiphospholipid antibody tests [66]. Bowles et al., in their case series of 34 patients with COVID-19 and a prolonged aPTT, found positive lupus anticoagulant assays in $31(91 \%)$ patients [67]. Thus, it is still unclear whether the observed coagulopathy is only a marker of the severity of the disease (as observed in septic shock) or whether the virus has a direct impact on the coagulation cascade $[63,68]$. Several investigations have tried to implicate coagulopathy indices in the survival prediction of patients with COVID-19 and posited the D-dimer level, the prothrombin time [3, 63], and thrombocytopenia [69] as potential prognostic factors. The suggested potential D-dimer cutoff points for predicting the severity of the disease are 0.5 [3], 2.4 [16], and $3 \mathrm{mg} / \mathrm{L}$ [63]. There is consensus regarding the role of Ddimer in patients with COVID-19, yet a single study reported that D-dimer was not a determinant in the clinical risk model for predicting the occurrence of critical illness [70]. The salient point is that D-dimer is generally accepted as an important factor in patients with COVID-19 [23]. Conversely, bleeding complications appear to be rare in this group of patients [71].

The coagulopathy described in COVID-19, in conjunction with a severe inflammatory response and hypoxia, may increase the risk of thrombotic events in patients with this infection [23]. A few reports have elaborated on the prevalence of different thrombotic complications in patients with COVID19. In a study conducted in three hospitals in the Netherlands, Klok et al. observed a $31 \%$ incidence rate of thrombotic complications, the majority of which were VTE (27\% of the patients), compared with arterial thrombotic events $(3.7 \%$ of the patients) [65]. Similarly, a high incidence of pulmonary emboli was observed by Poissy et al., who reported a rate of $20.6 \%$ of VTE in infected patients; in addition, the rate was twice that during the same time interval in the previous year [72]. It should be noted that the diagnosis of VTE might be very challenging in patients hospitalized for COVID-19. Apart from the fact that D-dimer is deemed inapplicable, the transfer of critically ill patients to imaging wards and then positioning them properly is by no means straightforward; as a result, the diagnostic process of considerable numbers of patients remains incomplete. Although not definitive, diagnostic measures such as right ventricular enlargement/ dysfunction on echocardiography or deep VTE in the lower limbs on ultrasound might be helpful toward the diagnosis of VTE. Controversy persists concerning the treatment of patients without a definite diagnosis, with various experts having suggested intermediate- to full-dose anticoagulation [23]. In patients with suspected pulmonary embolism at low bleeding risk, full-dose anticoagulation might be acceptable [73], whereas in patients with suspected pulmonary embolism at high bleeding risk, intermediate-dose anticoagulation could be considered [64, 73]. A half-therapeutic-dose regimen has been adopted as intermediate-dose anticoagulation in many centers [64]. Several trials are ongoing on the true value of each of these proposed regimens [74].

The symptom overlap between pulmonary emboli and acute respiratory disease in COVID-19 [75] and the aforementioned challenges in the diagnosis and treatment of pulmonary emboli in the pandemic call for an appropriate prophylaxis strategy. Tang et al. investigated the validity of the sepsisinduced coagulopathy score and the D-dimer level in the risk stratification of patients with COVID-19 with regard to VTE prophylaxis [24]. In their retrospective analysis on 449 patients hospitalized for severe COVID-19, there was no 28day mortality benefit among heparin (LMWH or UFH) users. However, in patients with a minimum sepsis-induced coagulopathy score of 4 or a D-dimer level greater than $3.0 \mu \mathrm{g} / \mathrm{mL}$, heparin prophylaxis significantly improved the 28-day mortality. Hence, Tang and colleagues recommended prophylaxis implementation based on risk stratification [24]. Other risk stratification tools (e.g., Caprini and IMPROVE) have also been suggested by some experts [23]. The International Society on Thrombosis and Haemostasis (ISTH) has offered a liberal recommendation, suggesting the administration of LMWH in all patients hospitalized for COVID-19 (including those who are not critically ill) who do not have contraindications (platelet count $\leq 25 \times 109 / \mathrm{L}$ or active bleeding) [71]. While there is currently a dearth of data suggesting this routine VTE prophylaxis, a case series by Klok et al. confirmed the high incidence of VTE $(27 \%)$ in patients hospitalized for COVID-19, which may be regarded as justification for the liberal approach. Some investigators have suggested a higher-than-routine i.e., "escalated" dose of VTE prophylaxis in patients in the ICU and especially patients with ARDS [64]. Successful experiences have been previously published [76] regarding the mentioned approach in patients with $\mathrm{H} 1 \mathrm{~N} 1$ complicated by ARDS. Accordingly, a meta-analysis of 9 trials showed that a minimum of $5000 \mathrm{U} / \mathrm{d}$ of LMWH succeeded in significantly decreasing the 28-day mortality among patients with ARDS (RR: $0.63,95 \% \mathrm{CI}: 0.41$ to 
0.96) [77]. An elevated level of D-dimer has been proposed as a marker for the benefit of an escalating regimen [64]. Additionally, mechanical prophylaxis has been suggested for patients with contraindications for pharmacological prophylaxis [23].

Notably, the importance of VTE prophylaxis has been recognized since the early days of the current pandemic. Wang et al., in their short report on 1026 patients hospitalized for COVID-19, showed that more than $40 \%$ of their patients obtained a minimum Padua Prediction Score of 4 (i.e., high risk for VTE) and only 7\% received appropriate treatment [78].

For patients on chronic treatment with anticoagulants, a switch to parenteral agents is recommended upon admission [23]. From the drug-interaction point of view, it is worth mentioning that warfarin can be used with COVID-19 medications with careful monitoring of the international normalized ratio (INR). According to the European Society of Cardiology (ESC) 2020 guideline, rivaroxaban and apixaban have fewer interactions with chloroquine/hydroxychloroquine than with dabigatran and edoxaban [19]. Still, with lopinavir/ritonavir, the use of rivaroxaban is contraindicated due to the increased risk of bleeding. Furthermore, edoxaban is not recommended because of the lack of evidence, the dose of apixaban should be halved, and dabigatran can be administered at a full dose with a 2-h gap of lopinavir/ritonavir [19, 25, 79].

\section{Conclusions}

It is vital to remember that cardiovascular complications remain the leading cause of death in the pandemic era. Indeed, cardiovascular complications are a common finding in COVID-19, and patients with underlying CVD are more susceptible to the infection. Pharmacotherapy, an integral component of patient care, should be adjusted in patients with active coronavirus infection based on their comorbidities, which may affect the drug choice and dosage. Drug interactions between cardiovascular medications and such antiviral agents as hydroxychloroquine and lopinavir/ritonavir constitute another issue deserving of special note. We herein sought to suggest appropriate therapies for the common cardiovascular problems of COVID-19, namely ACS, heart failure, myocarditis, arrhythmias, and VTE, based on the current literature. There is clearly a paucity of information on each of these complications at present, and further studies are needed to elucidate the best pharmacotherapy regimen in patients suffering from COVID-19.

\section{Compliance with Ethical Standards}

Conflict of Interest None declared.

\section{References}

1. https://www.who.int/dg/speeches/detail/whodirector-general-sopening-remarks-at-the-media-briefing-on-covid-19\%2D\%2D-11march-2020. Accessed 12 March 2020.

2. Zaim S, Chong JH, Sankaranarayanan V, Harky A. COVID-19 and multi-organ response. Curr Probl Cardiol. 2020;45(8):100618.

3. W-j G, Ni Z-y HY, et al. Clinical characteristics of coronavirus disease 2019 in China. N Engl J Med. 2020;382:1708-20.

4. Xiong T-Y, Redwood S, Prendergast B, Chen M. Coronaviruses and the cardiovascular system: acute and long-term implications. Eur Heart J. 2020;41(19):1798-800.

5. Ebinger JE, Shah PK. Declining admissions for acute cardiovascular illness: the Covid-19 paradox. J Am Coll Cardiol. 2020;1097(20):35394-8.

6. Picchianti Diamanti A, Rosado MM, Pioli C, Sesti G, Laganà B. Cytokine release syndrome in COVID-19 patients, a new scenario for an old concern: the fragile balance between infections and autoimmunity. Int J Mol Sci. 2020;21(9):3330.

7. Grein J, Ohmagari N, Shin D, et al. Compassionate use of remdesivir for patients with severe Covid-19. N Engl J Med. 2020.

8. Horby P, Lim WS, Emberson J, et al. Effect of Dexamethasone in Hospitalized Patients with COVID-19: Preliminary Report. medRxiv. 2020.

9. Li L, Zhang W, Hu Y, et al. Effect of convalescent plasma therapy on time to clinical improvement in patients with severe and lifethreatening COVID-19: a randomized clinical trial. JAMA. 2020.

10. Zhang H, Penninger JM, Li Y, Zhong N, Slutsky AS. Angiotensinconverting enzyme 2 (ACE2) as a SARS-CoV-2 receptor: molecular mechanisms and potential therapeutic target. Intensive Care Med. 2020;46:586-90.

11. Raizada MK, Ferreira AJ. ACE2: a new target for cardiovascular disease therapeutics. J Cardiovasc Pharm T. 2007;50(2):112-9.

12. Sodhi CP, Wohlford-Lenane C, Yamaguchi Y, et al. Attenuation of pulmonary ACE2 activity impairs inactivation of des-Arg9 bradykinin/BKB1R axis and facilitates LPS-induced neutrophil infiltration. Am J Physiol-Lung C. 2018;314(1):17-31.

13. Zheng Y-Y, Ma Y-T, Zhang J-Y, Xie X. COVID-19 and the cardiovascular system. Nat Rev Cardiol. 2020;17(5):259-60.

14. Chen L, Li X, Chen M, Feng Y, Xiong C. The ACE2 expression in human heart indicates new potential mechanism of heart injury among patients infected with SARS-CoV-2. Cardiovasc Res. 2020;116(6):1097-100.

15. Lippi G, Lavie CJ, Sanchis-Gomar F. Cardiac troponin I in patients with coronavirus disease 2019 (COVID-19): Evidence from a metaanalysis. Prog Cardiovasc Dis. 2020;S0033-0620(20):30055-4.

16. Huang C, Wang Y, Li X, et al. Clinical features of patients infected with 2019 novel coronavirus in Wuhan. China The Lancet. 2020;395(10223):497-506.

17. Shi S, Qin M, Shen B, et al. Association of cardiac injury with mortality in hospitalized patients with COVID-19 in Wuhan. JAMA Cardiol: China; 2020.

18. Wang D, Hu B, Hu C, et al. Clinical characteristics of 138 hospitalized patients with 2019 novel coronavirus-infected pneumonia in Wuhan. China JAMA. 2020;323(11):1061-9.

19. https://www.escardio.org/Education/COVID-19-and-Cardiology/ ESC-COVID-19-Guidance. Last updated on 10 June 2020.

20. Welt FG, Shah PB, Aronow HD, et al. Catheterization laboratory considerations during the coronavirus (COVID-19) pandemic: from ACC's interventional council and SCAI. J Am Coll Cardiol. 2020;75(18):2372-5.

21. Ibanez B, James S, Agewall S, et al. 2017 ESC guidelines for the management of acute myocardial infarction in patients presenting with ST-segment elevation. Kardiol Pol. 2018;76(2):229. 
22. Berwanger O, Lopes RD, Moia DD, et al. Ticagrelor versus clopidogrel in patients with STEMI treated with fibrinolysis: TREAT trial. J Am Coll Cardiol. 2019;73(22):2819-28.

23. Bikdeli B, Madhavan MV, Jimenez D, et al. COVID-19 and thrombotic or thromboembolic disease: implications for prevention, antithrombotic therapy, and follow-up. J Am Coll Cardiol. 2020;:S0735-1097(20):35008-7.

24. Tang N, Bai H, Chen X, Gong J, Li D, Sun Z. Anticoagulant treatment is associated with decreased mortality in severe coronavirus disease 2019 patients with coagulopathy. J Thromb Haemost. 2020;18(5):1094-9.

25. Dixon DL, Van Tassell BW, Vecchié A, et al. Cardiovascular considerations in treating patients with coronavirus disease 2019 (COVID-19). J Cardiovasc Pharmacol. 2020;75(5):359-67.

26. Grundy SM, Stone NJ, Bailey AL, et al. 2018 AHA/ACC/ AACVPR/AAPA/ABC/ACPM/ADA/AGS/APhA/ASPC/NLA/ PCNA guideline on the management of blood cholesterol: executive summary: a report of the American College of Cardiology/ American Heart Association task force on clinical practice guidelines. J Am Coll Cardiol. 2019;73(24):3168-209.

27. Newman CB, Preiss D, Tobert JA, et al. Statin safety and associated adverse events: a scientific statement from the American Heart Association. Arterioscler Thromb Vasc Biol. 2019;39(2):e38-81.

28. Giguère $\mathrm{P}$, Nhean $\mathrm{S}$, Tseng AL, Hughes CA, Angel JB. Getting to the heart of the matter: a review of drug interactions between HIV antiretrovirals and cardiology medications. Can J Cardiol. 2019;35(3):326-40.

29. Fang L, Karakiulakis G, Roth M. Are patients with hypertension and diabetes mellitus at increased risk for COVID-19 infection? Lancet Respir Med. 2020;8(4):e21.

30. Kuba K, Imai Y, Rao S, et al. A crucial role of angiotensin converting enzyme 2 (ACE2) in SARS coronavirus-induced lung injury. Nat Med. 2005;11(8):875-9.

31. Mancia G, Rea F, Ludergnani M, Apolone G, Corrao G. Reninangiotensin-aldosterone system blockers and the risk of Covid-19. N Engl J Med. 2020.

32. Reynolds HR, Adhikari S, Pulgarin C, et al. Renin-angiotensinaldosterone system inhibitors and risk of Covid-19. N Engl J Med. 2020.

33. Mehra MR, Desai SS, Kuy S, Henry TD, Patel AN. Cardiovascular disease, drug therapy, and mortality in COVID-19. N Engl J Med. 2020.

34. Bozkurt B, Kovacs R, Harrington B. Joint HFSA/ACC/AHA statement addresses concerns re: using RAAS antagonists in COVID19. J Card Fail. 2020;26(5):370.

35. Cardiology ESo. Position statement of the ESC Council on hypertension on ACE-inhibitors and angiotensin receptor blockers. ESC. 2020.

36. Mehra MR, Ruschitzka F. COVID-19 illness and heart failure: a missing link? JACC Heart Fail. 2020;8(6):512-4.

37. Dong N, Cai J, Zhou Y, Liu J, Li F. End-stage heart failure with COVID-19: strong evidence of myocardial injury by 2019-nCoV. JACC Heart Fail. 2020;S2213-1779(20):30200-6.

38. Caforio AL, Pankuweit S, Arbustini E, et al. Current state of knowledge on aetiology, diagnosis, management, and therapy of myocarditis: a position statement of the European Society of Cardiology Working Group on myocardial and pericardial diseases. Eur Heart J. 2013;34(33):2636-48.

39. Inciardi RM, Lupi L, Zaccone G, et al. Cardiac involvement in a patient with coronavirus disease 2019 (COVID-19). JAMA Cardiol. 2020.

40. Hu H, Ma F, Wei X, Fang Y. Coronavirus fulminant myocarditis saved with glucocorticoid and human immunoglobulin. Eur Heart J. 2020.
41. Tavazzi G, Pellegrini C, Maurelli M, et al. Myocardial localization of coronavirus in COVID-19 cardiogenic shock. Eur Heart Fail. 2020;22(5):911-5.

42. Alhazzani W, Møller MH, Arabi YM, et al. Surviving Sepsis campaign: guidelines on the management of critically ill adults with coronavirus disease 2019 (COVID-19). Intensive Care Med. 2020;46:854-87.

43. Poston JT, Patel BK, Davis AM. Management of critically ill adults with COVID-19. JAMA. 2020;323(18):1839-41.

44. Frustaci A, Chimenti C, Calabrese F, Pieroni M, Thiene G, Maseri A. Immunosuppressive therapy for active lymphocytic myocarditis: virological and immunologic profile of responders versus nonresponders. Circulation. 2003;107(6):857-63.

45. Paul J-F, Charles P, Richaud C, Caussin C, Diakov C. Myocarditis revealing COVID-19 infection in a young patient. Eur Heart J Cardiovas Imaging. 2020:jeaa107.

46. Dabbagh MF, Aurora L, D'Souza P, Weinmann AJ, Bhargava P, Basir MB. Cardiac tamponade secondary to COVID-19. Case Reports: JACC; 2020.

47. Deftereos SG, Giannopoulos G, Vrachatis DA, et al. Effect of colchicine vs standard care on cardiac and inflammatory biomarkers and clinical outcomes in patients hospitalized with coronavirus disease 2019: the GRECCO-19 randomized clinical trial. JAMA Netw Open. 2020;3(6):e2013136.

48. Driggin E, Madhavan MV, Bikdeli B, et al. Cardiovascular considerations for patients, health care workers, and health systems during the coronavirus disease 2019 (COVID-19) pandemic. J Am Coll Cardiol. 2020;75(18):2352-71.

49. Bansal M. Cardiovascular disease and COVID-19. Diabetes Metab Syndr. 2020;14(3):247-50.

50. Singh S, Desai R. COVID-19 And New Onset Arrhythmia. Journal of Arrhythmia. 2020:joa3.

51. Lazzerini PE, Boutjdir M, Capecchi PL. COVID-19, arrhythmic risk and inflammation: mind the gap! Circulation. 2020.

52. Zeitlinger M. Pharmacokinetics-pharmacodynamics of antiviral agents used to treat SARS-CoV-2 and their potential interaction with drugs and other supportive measures: a comprehensive review by the PK/PD of anti-Infectives study Group of the European Society of antimicrobial agents. 2020.

53. Giudicessi JR. Urgent guidance for navigating and circumventing the QTc prolonging and Torsadogenic potential of possible pharmacotherapies for COVID-19. Mayo Clin Proc. 2020;6:1213-21.

54. Mehra MR, Desai SS, Ruschitzka F, Patel AN. Hydroxychloroquine or chloroquine with or without a macrolide for treatment of COVID-19: a multinational registry analysis. Lancet. 2020;S0140-6736(20):31180-6.

55. Tisdale JE, Jaynes HA, Kingery JR, et al. Development and validation of a risk score to predict QT interval prolongation in hospitalized patients. Cir Cardiovasc Qual. 2013;6(4):479-87.

56. Haugaa KH, Bos JM, Tarrell RF, Morlan BW, Caraballo PJ, Ackerman MJ. Institution-wide QT alert system identifies patients with a high risk of mortality. Mayo Clin Proc. 2013;88(4):315-25.

57. Simpson TF, Kovacs RJ, Stecker EC. Ventricular arrhythmia risk due to Hydroxychloroquine-azithromycin treatment for COVID19. Ame Coll Cardiol. 2020.

58. Roden DM, Harrington RA, Poppas A, Russo AM. Considerations for drug interactions on QTc in exploratory COVID-19 (coronavirus disease 2019) treatment. Circulation. 2020.

59. Sapp JL, Alqarawi W, MacIntyre CJ, et al. Guidance on minimizing risk of drug-induced ventricular arrhythmia during treatment of COVID-19: a statement from the Canadian Heart Rhythm Society. Can J Cardiol. 2020.

60. Wu C-I, Postema PG, Arbelo E, et al. SARS-CoV-2, COVID-19 and inherited arrhythmia syndromes. Heart Rhythm. 2020. 
61. Jeevaratnam K. Chloroquine and hydroxychloroquine for COVID19: implications for cardiac safety. Eur Heart J Cardiovasc Pharmacother. 2020:pvaa041.

62. Vidovich MI. Transient Brugada-like electrocardiographic pattern in a patient with COVID-19. Case Reports: JACC; 2020.

63. Tang N, Li D, Wang X, Sun Z. Abnormal coagulation parameters are associated with poor prognosis in patients with novel coronavirus pneumonia. J Thromb Haemost. 2020;18(4):884-7.

64. Connors JM, Levy JH. COVID-19 and its implications for thrombosis and anticoagulation. Blood. 2020;135(23):2033-40.

65. Klok FA, Kruip MJHA, van der Meer NJM, et al. Incidence of thrombotic complications in critically ill ICU patients with COVID-19. Thromb Res. 2020;191:145-7.

66. Zhang Y, Xiao M, Zhang S, et al. Coagulopathy and antiphospholipid antibodies in patients with Covid-19. N Engl J Med. 2020;382(17):e38.

67. Bowles L, Platton S, Yartey N, et al. Lupus anticoagulant and abnormal coagulation tests in patients with Covid-19. N Engl J Med. 2020.

68. Iba T, Levy JH, Warkentin TE, et al. Diagnosis and management of sepsis-induced coagulopathy and disseminated intravascular coagulation. J Thromb Haemost. 2019;17(11):1989-94.

69. Lippi G, Plebani M, Henry BM. Thrombocytopenia is associated with severe coronavirus disease 2019 (COVID-19) infections: a meta-analysis. Clin Chim Acta. 2020.

70. Liang W, Liang H, Ou L, et al. Development and validation of a clinical risk score to predict the occurrence of critical illness in hospitalized patients with COVID-19. JAMA Intern Med. 2020.

71. Thachil J, Tang N, Gando S, et al. ISTH interim guidance on recognition and management of coagulopathy in COVID-19. J Thromb Haemost. 2020;18(5):1023-6.
72. Poissy J, Goutay J, Caplan M, et al. Pulmonary embolism in COVID-19 patients: awareness of an increased prevalence. Circulation. 2020.

73. Obi AT, Barnes GD, Wakefield TW, Eliason JL, Arndt E, Henke PK. Practical diagnosis and treatment of suspected venous thromboembolism during COVID-19 pandemic. J Vasc Surg-Venous L. 2020.

74. Bikdeli B, Madhavan MV, Gupta A, et al. Pharmacological agents targeting Thromboinflammation in COVID-19: review and implications for future research. Thromb Haemost. 2020.

75. Danzi GB, Loffi M, Galeazzi G, Gherbesi E. Acute pulmonary embolism and COVID-19 pneumonia: a random association? Eur Heart J. 2020;41(19):1858.

76. Obi AT, Tignanelli CJ, Jacobs BN, et al. Empirical systemic anticoagulation is associated with decreased venous thromboembolism in critically ill influenza a H1N1 acute respiratory distress syndrome patients. J Vasc Surg-Venous L. 2019;7(3):317-24.

77. Li J, Yang B, Wang H. Low-molecular-weight heparin treatment for acute lung injury/acute respiratory distress syndrome: a metaanalysis of randomized controlled trials. Int J Clin Exp Med. 2018;11(2):414-22.

78. Wang T, Chen R, Liu C, et al. Attention should be paid to venous thromboembolism prophylaxis in the management of COVID-19. Lancet Haematol. 2020;7(5):e362-e3.

79. Wiggins BS, Dixon DL, Neyens RR, Page RL II, Gluckman TJ. Select drug-drug interactions with direct oral anticoagulants: JACC review topic of the week. J Am Coll Cardiol. 2020;75(11):1341-50.

Publisher's Note Springer Nature remains neutral with regard to jurisdictional claims in published maps and institutional affiliations. 